Nodocentes: las tutorías como dispositivos de acompañamiento académico

Elizabeth F. Sanfilippo, Carlos A. Vázquez

Revista ES (en y sobre Educación Superior)

Vol.1, N¹-2 / Fecha de publicación: 27/12/2021

e-ISSN: 2718-6539

https://revistas.unlp.edu.ar/ES/index

IIES - Facultad de Odontología

DOI: https://doi.org/10.24215/27186539e035

\title{
Nodocentes: las tutorías como dispositivos de acompañamiento académico
}

Nodocentes: tutoring as academic accompaniment devices

Nodocentes: tutoria como dispositivo de acompanhamento acadêmico

Licenciada Elizabeth F. Sanfilippo

Facultad de Arquitectura y Urbanismo -UNLP

lizysanfilippo@gmail.com

Carlos A. Vázquez

\section{Resumen}

La formación profesional del plantel de trabajadores puede ser un objetivo a alcanzar por distintas organizaciones. Pero si esa formación puede brindarse desde la propia organización, entonces no sólo se avanza en mejorar las 
capacidades de gestión del personal jerárquico y de los posibles sustitutos, sino que se logra una noción más acabada del conjunto de la Universidad por parte del estudiante. Por lo tanto, con ese conocimiento se puede pensar en la posibilidad de colaborar con iniciativas que mejoren o resuelvan problemáticas administrativas y de gestión en áreas carentes de estructura Nodocente, ya sea por su reciente creación o por no formar parte de los espacios tradicionalmente ocupados por este tipo de trabajadores.

El esfuerzo que realizan los y las estudiantes de la Licenciatura en Gestión de Recursos para Instituciones Universitarias, en su mayoría adultos, con familia a cargo y treinta y cinco horas semanales de labor en Facultades 0 Dependencias universitarias, no debe minimizarse. El costo de oportunidad de este universo estudiantil es muy elevado y, en consecuencia, la detección temprana de las problemáticas que conlleva el tránsito por la carrera de la Licenciatura permitiría tomar decisiones respecto a las estrategias que le permitan al estudiante superar dificultades áulicas y de planificación familiar, entre otras, tendiendo a disminuir los índices de desgranamiento que han sido observados en las primeras cuatro cohortes que desde el 2006 continúan hasta la actualidad, ampliando el número de graduados.

\section{Abstract}

Vocational training on the workers' campus can be an objective to be achieved by different organizations. But if this training can be provided from the organization itself, then not only is progress being made in improving the management capabilities of hierarchical staff and potential substitutes, but a more finished notion of the university as a whole is achieved by the student. Therefore, with this knowledge one can think of the possibility of collaborating 
with initiatives that improve or solve administrative and management problems in areas lacking a Nodocente structure, either because of its recent creation or because it is not part of the spaces traditionally occupied by this type of worker. The efforts made by students of the Bachelor's Degree in Resource Management for University Institutions, mostly adults, with family in charge and thirty-five hours per week of work in Colleges or University Dependencies, should not be minimized. The opportunity cost of this student universe is very high and, consequently, the early detection of the problems involved in transit through the Bachelor's degree would allow decisions to be made regarding strategies that allow the student to overcome aulic and family planning difficulties, among others, tending to decrease the rates of degreasing that have been observed in the first four cohorts that continue from 2006 to the present day, expanding the number of graduates.

\section{Resumo}

A qualificação profissional da força de trabalho pode ser um objetivo a ser alcançado por diferentes organizações. Mas se este treinamento pode ser fornecido pela própria organização, então não só se progride no aprimoramento das habilidades de gestão do pessoal hierárquico e seus possíveis substitutos, mas uma noção mais completa da Universidade como um todo é alcançada pelo aluno. Assim, com este conhecimento, é possível pensar na possibilidade de colaborar com iniciativas que melhorem ou resolvam problemas administrativos e de gestão em áreas sem estrutura Nodocent, quer pela sua criação recente, quer por não fazerem parte dos espaços tradicionalmente ocupados. por este tipo de trabalhador. 
O esforço realizado pelos alunos do Curso de Licenciatura em Gestão de Recursos para Instituições Universitárias, na sua maioria adultos, com família a cargo e trinta e cinco horas semanais de trabalho em Faculdades ou Departamentos Universitários, não deve ser minimizado. O custo de oportunidade desse universo discente é muito alto e, consequentemente, a detecção precoce dos problemas que o trânsito pelo bacharelado acarreta permitiria tomar decisões quanto às estratégias que permitem ao aluno superar as dificuldades de sala de aula e de planejamento familiar, entre outras, tendendo a diminuir os índices de descasque observados nas quatro primeiras coortes que de 2006 continuam até os dias atuais, aumentando o número de egressos.

\section{Palabras clave}

Tutoría, Dispositivo, Nodocente, Acompañamiento académico.

\section{Keywords}

Tutoring, Device, Nodocente, Academic Accompaniment.

\section{Palavras chave}

Tutoria, Dispositivo, Nodocente, Acompanhamento acadêmico.

\section{Desarrollo}

El presente artículo fue concebido como parte de la propuesta que oportunamente desarrolláramos para el Instituto de Investigaciones en Educación Superior (IIES), en el marco de la tesina final de la Carrera de Especialización en Educación Superior, donde nos propusimos analizar una 
propuestas viable para evitar el desgranamiento en una de las principales opciones para la formación profesional del plantel de trabajadores de la Universidad Nacional de La Plata (UNLP), ya que consideramos que éste puede ser un objetivo a alcanzar, como ocurre con distintas organizaciones.

El factor diferencial es que, si esa formación puede brindarse desde la propia organización, entonces no sólo se avanza al mejorar las capacidades de gestión del personal jerárquico y sus posibles sustitutos, sino que se logra una noción más acabada del conjunto de la institución (en nuestro caso, la UNLP) por parte del estudiante que recibe dicha capacitación. Por lo tanto, ese conocimiento le puede permitir colaborar con iniciativas que luego mejoren o resuelvan problemáticas administrativas y de gestión en áreas carentes de una estructura Nodocente, ya sea por su reciente creación o por no formar parte de los espacios tradicionalmente ocupados por este tipo de trabajadores.

En ese marco, apuntamos a analizar el esfuerzo que realizan los y las estudiantes de la Licenciatura en Gestión de Recursos para Instituciones Universitarias, una matrícula compuesta en su mayoría por trabajadores y trabajadoras en su adultez, con cargas familiares repartidas con las treinta y cinco horas semanales de labor en sus Facultades o Dependencias universitarias, aspectos diferenciales que no deben minimizarse.

Estas características distintivas conllevan un costo de oportunidad para este universo estudiantil muy elevado y, en consecuencia, la detección temprana de estas potenciales problemáticas durante el tránsito en la Licenciatura, les permitiría tomar decisiones estratégicas que, entendemos, les permitirán superar dificultades áulicas y de planificación familiar, entre otras, tendiendo a disminuir los índices de desgranamiento que detallaremos, y que fueron observados en las primeras cuatro cohortes que ha tenido esta Carrera desde 
su creación en 2006. Si esto ocurre, consecuentemente se podrá aumentar el número de graduados que la carrera ostenta actualmente.

Es por ello que, con el objetivo de promover el tránsito y egreso de los estudiantes de la Licenciatura en Gestión de Recursos para Instituciones Universitarias (LGRIU), proponemos diseñar una estrategia que permita promover un dispositivo académico de acompañamiento continuo al trayecto formativo de los y las Nodocentes que cursan la carrera, para favorecer la disminución de los índices de desgranamiento y repitencia que se producen en cada cohorte. Cabe mencionar, respecto a la repitencia, que quien desaprueba una asignatura debe esperar cuatro años para volver a cursarla, dado que actualmente se dictan a cohorte cerrada.

La implementación de la LGRIU en la UNLP fue posible en un marco colaborativo interinstitucional en el que intervienen, por un lado, la Dirección de Capacitación Laboral (dependiente de la Prosecretaría de Planificación y Gestión Laboral de la Presidencia de la UNLP); la Facultad de Ciencias Jurídicas y Sociales de la UNLP; y la Secretaría de Capacitación de la Asociación de Trabajadores Nodocentes de la Universidad Nacional de La Plata (ATULP), que interviene en la ejecución de la carrera.

En este marco, la LGRIU no puede dejar de perseguir el objetivo que enunciará el Doctor Fernando Tauber en el Proyecto Institucional 2018-2022, por lo que debe tener como meta

“...ser firmemente inclusiva y entender a la educación como una línea ininterrumpida e ininterrumpible, que no debe cortarse por una falla del sistema que frene el proceso de toma de conocimientos de aquel que quiere seguir sumándolos, por un fracaso o deficiencia circunstancial. Si un estudiante no logra avanzar a un estadio superior en el proceso de su 
formación, pero tiene la firme voluntad de resolver sus déficits esforzándose en sus estudios, la universidad debe darle siempre esa oportunidad. No puede dejarlo flotando en un limbo que no le permita ni retroceder ni avanzar. $\mathrm{Y}$ esto debe suceder desde el ingreso hasta la finalización de sus estudios". ${ }^{1}$ (Tauber, 2018, p.5)

\section{Antecedentes de la Licenciatura en Gestión de Recursos para Instituciones Universitarias}

A mediados de la década del ' 90 , tiempos en los que se comenzaba a negociar un Convenio Colectivo de Trabajo (CCT) para los y las Nodocentes de las Universidades Nacionales, surgieron algunos consensos básicos entre los que se plasmó el derecho laboral a recibir capacitación de los trabajadores y las trabajadoras del sector. Con ese importante sustento, las representaciones gremiales plasmaron la necesidad de crear carreras de pre-grado que, bajo la forma de la Tecnicatura en Gestión Universitaria, siguieran los lineamientos del Convenio.

En el caso de la UNLP, dicha Tecnicatura recaló en la estructura académica de la Facultad de Ciencias Jurídicas y Sociales, con una malla curricular diseñada a corte cerrada para ser cursadas en tres años.

Los primeros datos analizados (que pueden observarse en el Cuadro 1 y los Gráficos 1 y 2), responden a las primeras dos cohortes de dicha Tecnicatura, en las que observamos que el desgranamiento aumentó entre cohortes hasta alcanzar al $60 \%$ de los y las estudiantes inscriptas en 2009, mientras que la

\footnotetext{
1 Tauber, F. (2018) "Pensar la Universidad. Proyecto Institucional de la Universidad Nacional de La Plata 2018-2022".
} 
tasa de graduación a tiempo (al completar los tres años iniciales sin recursadas) ronda el $40 \%$.

CUADRO 1

\begin{tabular}{|c|c|c|c|}
\hline & Inscriptos & Cursaron & Egresaron \\
\hline $\mathbf{2 0 0 6}$ & 130 & 94 & 55 \\
\hline $\mathbf{2 0 0 9}$ & 101 & $\mathrm{~s} / \mathrm{d}$ & 40 \\
\hline
\end{tabular}

Fuente: Secretaría de Capacitación y Cultura ATULP

(2012).

Estos índices fueron presentados en abril de 2012 al "Primer Congreso de Graduados y Estudiantes de Tecnicaturas y Licenciaturas en Administración y Gestión Universitaria" realizado en la sede de la Federación Argentina de Trabajadores de las Universidades Nacionales (FATUN). Allí, los expositores destacaron que en las carreras semejantes que dictaron las Universidades de Catamarca, Tucumán y Córdoba, donde las tasas de deserción eran mayores y las de egreso no superaban el 7\%, habían comenzado a implementar sistemas de tutorías para sus estudiantes. Estos sistemas, en consonancia con lo que postulan García y otros en su artículo sobre el impacto de los programas de tutorías, "fortalece el desarrollo formativo de los estudiantes y estimula el desarrollo de habilidades intelectuales necesarias para elevar el desempeño académico de los alumnos"2, mejorando las tasas de permanencia y egreso. Es por ello que consideramos que establecer un sistema de tutorías en la Licenciatura dentro de una Unidad Pedagógica que dé seguimiento a esta propuesta, permitirá

${ }^{2}$ García, R. I., Cuevas, O., Vales, J. J. y Cruz, I. (2012). Impacto del Programa de Tutoría en el desempeño académico de los alumnos del Instituto Tecnológico de Sonora. Revista Electrónica de Investigación Educativa, 14(1), 106-121. 
mejorar los índices de graduación que se obtuvieron con las dos primeras cohortes de la Tecnicatura, y que pueden observarse en los Gráficos 1 y 2.

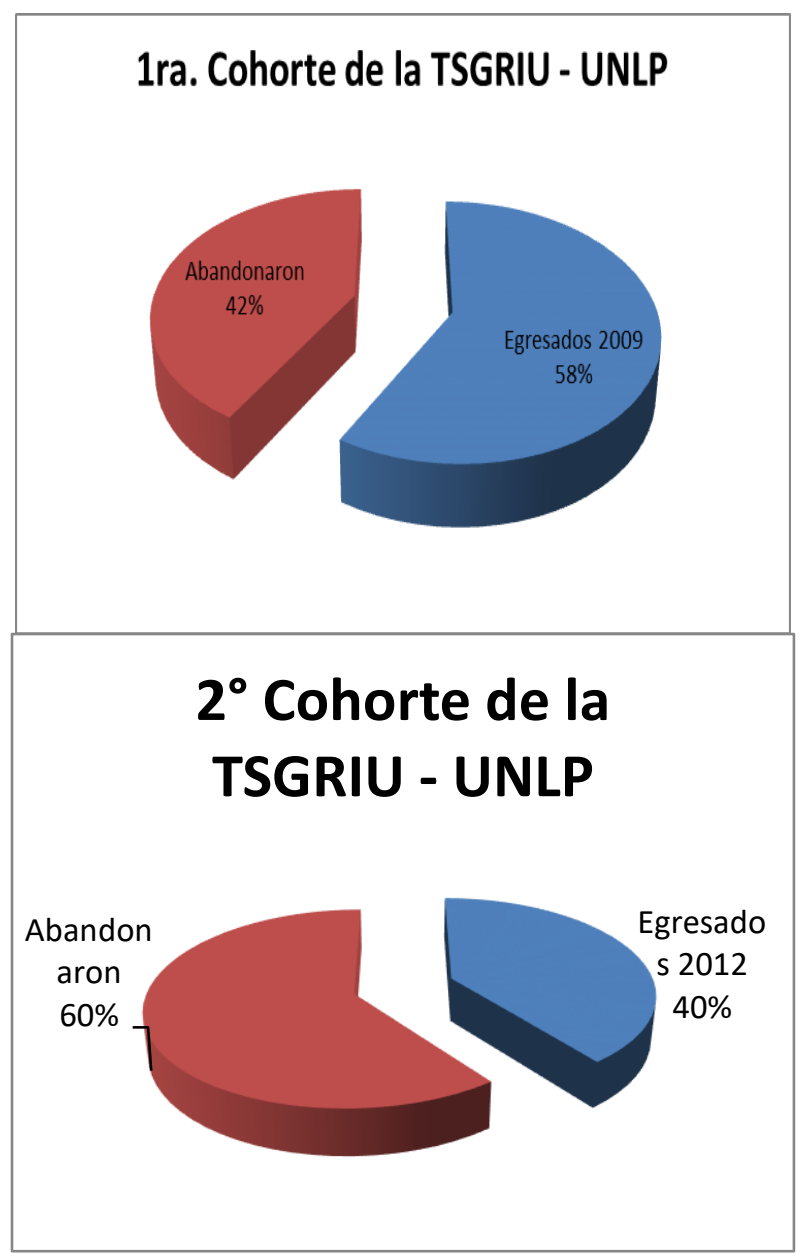

Nota: El porcentaje de graduación para la $1^{\circ}$ Cohorte aumentó en los años siguientes, cuando 39 de sus estudiantes lograron retomar y culminar sus estudios.

Cabe destacar que la literatura analiza a los sistemas de tutorías según se trate de las destinadas a los sistemas de educación universitaria presenciales o a los diseñados para su dictado a distancia. A su vez, puede distinguirse entre 
tutorías académicas y disciplinares, donde las primeras plantean la necesidad de distinguir entre tutores que acompañen al estudiante durante su carrera (algunas distinguen tutores para primeros años y otros para superiores), mientras que en las segundas deben tener experticia sobre una o más áreas disciplinares.

Los estudios recientes también distinguen la manera de pensar la estrategia de constituir espacios de seguimiento institucional de los estudiantes, a través de tutores que trabajen en forma sincrónica o asincrónica con ellos, lo que a su vez puede implicar la necesidad de una mediación tecnológica entre el tutor y el estudiante (o grupo de ellos) que, en el caso de la educación a distancia, es insoslayable.

Las definiciones atienden también a las ventajas que implica que los tutores sean estudiantes avanzados (pares), o bien docentes con experticia en comportamientos, como ser los psicólogos, los psicopedagogos y los trabajadores sociales. A falta de otros estudios indagatorios y ante estas dos perspectivas sobre las tutorías, pienso que quienes ya han egresado y/o son compañeros y compañeras en la planta permanente en los lugares de trabajo de los estudiantes de la LGU, pueden incluirse dentro de este último grupo, ya que son capaces de generar empatía y la confianza necesaria para articular con este tipo de estrategias.

Consideramos que, sin dudas, la tutoría académica y la tutoría de pares serán de utilidad para mejorar la performance de los y las estudiantes de la LGRIU de la UNLP. En tanto que la disciplinar, que puede resultar de cierta eficiencia, dependerá de los proyectos que en cada ocasión se aprueben para el dictado de las diversas asignaturas. 
Ahora bien: los estudiantes de la carrera de LGRIU son trabajadores que desean progresar en su carrera Nodocente a través de una formación de grado orientada al conocimiento profundo de los ámbitos de gestión universitarios, así como de sus posibles intervenciones dentro de sus espacios laborales, ya sea mejorando un proceso propio de su oficina o espacio de trabajo, o en algo de un alcance mayor, como proponer y/o ejecutar proyectos que favorezcan la mejora continua de un Área en su Facultad o Dependencia.

En algunos casos, los estimula a graduarse el incremento salarial que les significa terminar la carrera, en particular cuando trabajan en estructuras que no cuentan con posibilidades de ascenso en el escalafón en un futuro cercano. En cambio, otros estudiantes valoran más las posibilidades relativas que este título les otorgaría frente a otros participantes en potenciales concursos Nodocentes.

Por su parte, la UNLP también encuentra sus propias necesidades, ya que una mayor tasa de graduación le otorga una mayor profesionalización de sus planteles de trabajadores y le asegura contar con personal especialmente calificado para brindar soporte a los servicios de apoyo académico, de extensión, de investigación y de transferencia, ya que el personal contaría con un conocimiento integral de la organización.

Cabe destacar, además, que la matrícula de las viejas Tecnicaturas, así como de la actual LGRIU, están orientadas a trabajadores de Universidades Nacionales, por lo que su matrícula potencial tiene un universo finito que asciende a 51.542 Nodocentes (de acuerdo con los datos de la Secretaría de Políticas Universitarias) ${ }^{3}$ que, en promedio, cuentan con veinte años de

${ }^{3}$ Sobre los datos obtenidos desde el Sistema de consulta de estadísticas universitarias de la Secretaría de Políticas Universitarias de la Nación - SPU 
servicio. Esto implica que los estudiantes de estas carreras tienen, en promedio entre 30 y 40 años de edad y alguna experiencia áulica en la Educación Superior, aunque también contempla un alto grado de dispersión etaria, lo que ha permitido a Nodocentes de hasta 60 años de edad haber logrado su graduación.

Esta característica distintiva en la heterogeneidad de edades de la matrícula de las TGU, se complementa con la igualmente heterogénea experiencia laboral y de constitución familiar de cada uno de los estudiantes. Es en este punto donde la visión de una tutoría como estrategia para aumentar el grado de terminalidad de los estudios encuentra sustento en el esfuerzo institucional por sostener y acompañar el tránsito académico que permita la profesionalización de la actividad de sus Nodocentes, cuidando, acompañando y orientándolos como estudiantes, para que puedan aprovechar al máximo sus potencialidades.

Por otro lado, se torna necesario caracterizar aquí a los estudiantes de la Carrera, dado que la particularidad se da en que ésta se dicta para Nodocentes pertenecientes a la UNLP, quienes cuentan con un trabajo estable en relación de dependencia con la institución y, por ende, con una carga horaria de al menos 35 horas semanales en una alta diversidad de funciones y actividades laborales. Los datos provenientes de la Dirección de Capacitación Laboral demuestran que a lo largo de las cuatro cortes cerradas ya se ha alcanzado una tasa de graduación del $45 \%$, es decir que el índice de desgranamiento es lo suficientemente significativo como para proponer intervenciones tendientes a disminuir las causales de abandono de la Carrera.

consultada el 30 de abril de 2020. Disponible en


Es importante entender qué está pasando en el resto del sistema universitario con los TGUs y LGUs y, por ello, debo resaltar también la segunda cuestión a atender son las características propias del modelo presencial que tiene actualmente la UNLP. A saber, la LGRIU funciona a ciclo cerrado, con una sola banda horaria (vespertina), y con dictado teórico-práctico en las instalaciones de la sede gremial de ATULP que, además, se constituyó como mecanismo de divulgación de la carrera. Atendiendo entonces a la necesidad de sostener el proceso de formación, se vuelve prioritario realizar un análisis profundo de las motivaciones que llevaron a que aquellos Nodocentes inscriptos en las TGU decidiesen no iniciar sus estudios luego de haber registrado sus inscripciones, o bien que hubieran decidido abandonarlos una vez iniciados.

Las estadísticas que he realizado a partir de encuestas relevadas en $2012^{4}$ respecto a esta temática, permiten observar algunas de estas razones. A saber:

- Falta de motivación en el espacio laboral (en especial aquellos que contaban ya con algún grado de formación universitaria, aunque no hubieran alcanzado la titulación)

- Dificultades de planificación laboral y familiar (al no contar la Carrera con bandas horarias para cursar, muchos esperaban contar con la posibilidad de cursar en horario laboral o sin compensación de carga horaria)

- Otras dificultades ocasionadas por llevar más de diez años sin continuar estudios formales rigurosos.

4 Trabajo de investigación propio para la asignatura Epistemología y Metodologías de la Carrera de Licenciatura en Gestión de Recursos para Instituciones Universitarias/ FDyCS- UNLP se representan las cifras en los Anexos III y IV 
- La formación laboral era muy específica (como ocurre con los Nodocentes del agrupamiento Técnico Profesional) o muy básica (como ocurre en el caso de algunos empleados del agrupamiento de Servicios Generales y Mantenimiento, cuyo requisito por CCT es haber finalizado los estudios primarios).

Todos estos condicionantes fueron atendidos oportunamente en otras Universidades Nacionales que planificaron distintas estrategias para favorecer la retención de la matricula inicial. Para ello, por ejemplo, la Universidad Nacional de Tucumán (que cuenta con una planta permanente de dimensiones similares a la de la UNLP) resolvió que los docentes sean designados a propuesta de un jurado integrado por representantes de la gestión de la Universidad y la representación gremial. Además, estos docentes deben preparar y dictar sus clases, y proponer horarios de encuentro extracurriculares con los estudiantes de las materias a su cargo, para realizar así un seguimiento tutorial previo a la presentación de sus actividades o trabajos prácticos, lo que fortaleció el vínculo docente-estudiante.

Por su parte, en 2012 la Universidad Nacional de Catamarca contaba con más del $10 \%$ de su planta permanente ya graduada de su TGU. Esta situación se sumó allí a la modalidad de tecnicatura que fue adoptada por dicha Universidad, donde los docentes dictaron clases por extensión, y no por concurso ni retribución especial; todo esto conjugado con una estrategia de acompañamiento tutorial para la segunda, la tercera y la cuarta cohorte, a propuesta del Área de Capacitación de la Universidad, también integrada por personal docente y gremial. Allí los tutores pares fueron designados por la Universidad de Catamarca como Ayudantes Diplomados ad-honorem, con la particularidad de ser egresados de las primeras cohortes. 
El tercer caso a distinguir es el de la Universidad Nacional de Córdoba, con cursadas semi-presenciales que tienen soporte a través de la plataforma PROED. Esta exigía al docente una respuesta personalizada y asincrónica, a distancia. Para este tipo de tecnicatura fue necesario preparar a los inscriptos en el uso de tecnologías informáticas indispensables para que pudieran aprovechar al máximo la transmisión de contenidos y la comunicación docenteestudiante, lo que se concretó a través de seminarios específicos y con tutores que siguieron acompañando la trayectoria curricular. Esta modalidad busco dar respuesta a algunas variables de deserción, especialmente a las relacionadas con la imposibilidad de planificar la carga horaria áulica indispensable que un conjunto de Nodocentes tenían en la modalidad presencial, y de otro grupo cuya distancia física respecto a los lugares donde se dictaba la tecnicatura les impedía hacer el esfuerzo para formarse. Este último aspecto no solo es observable en la Universidad Nacional de Córdoba, ya que es general al sistema universitario nacional, donde se repite la dispersión geográfica de las unidades académicas y dependencias que las conforman.

\section{Reflexiones finales}

Las experiencias existentes en otras instituciones universitarias demuestran que los resultados alcanzados mediante políticas de tutorías sostenidas han sido exitosas en el universo de su aplicación, donde la información para la toma de decisiones es rápidamente analizada y utilizada en un proceso de continua retroalimentación, lo que mejora tanto el quehacer docente como la gestión administrativa, pero siempre centrada en la dimensión personalizada de la educación. Esto genera un fuerte vínculo del estudiante con la institución que lo forma y acompaña. De este modo, el esfuerzo económico de la Universidad no 
debería constituirse en un impedimento para la implementación de esta estrategia y la estructura que surja de las necesidades de acompañamiento en término de los acuerdos políticos alcanzados por los múltiples actores intervinientes, ya que brindará como resultado un aumento en la profesionalización de la planta permanente de la UNLP, generando beneficios tanto para el trabajador como para la Institución.

Por último, el aumento del índice de graduación no sólo se constituye en la contracara de la disminución del índice antagónico de desgranamiento, sino que genera masa crítica para pensar el siguiente nivel de formación. Es decir, la graduación en la Licenciatura es contar con la posibilidad de que ellos comiencen a recorrer formaciones de postgrado (dentro de la propia UNLP) orientados a líneas de gestión general o específica, como en algunos casos ya se ha dado.

\section{Bibliografía}

- Abate S. y Orellano V. (2015). Notas sobre el curriculum universitario, prácticas profesionales y saberes en uso. en Trayectorias Universitarias. Vol 1. No 1 . UNLP. ISSN 2469-0090. Disponible en: http://revistas.unlp.edu.ar/TrayectoriasUniversitarias/article/view/2307

- ANUIES (2010). La tutoría académica y la calidad de la educación. Programa de Institucional de Tutorías Académicas. Guadalajara, México. Disponible en: http://www.upn213.mx/archivos/Tutorias\%20Educacion\%20Superior\%20 -\%20Tutoria\%20Guadalajara.pdf

- Aparicio, M. (2008) "La deserción universitaria y su relación con factores motivacionales." Rev. Diálogos Pedagógicos, Año VI N 11, Pag. 11-26. 
- Ariza Ordóñez, G. I. y Ocampo Villegas, H. B. (2005). El acompañamiento tutorial como estrategia de la formación personal y profesional: un estudio basado en la experiencia en una institución de educación superior. Universitas Psychologica, 4(1),31-41.

Disponible en: https://www.redalyc.org/articulo.oa?id=64740104

- Biggs J. (2005) Calidad del aprendizaje universitario. Madrid. España. Narcea, S. A. Ediciones ISBN.: 84-277-1398-3

- Capelari, M. I. (2014). Las políticas de tutoría en la Educación Superior: Génesis, trayectorias e impactos en Argentina y México. Revista Latinoamericana de Educación Superior, 5, 41-54.

Disponible en: http://www.saece.com.ar/relec/revistas/5/mon3.pdf

- Carli, S. (2012) El estudiante universitario. Hacia una historia del presente de la educación pública. Buenos Aires Argentina. Siglo XXI Editores

- Carli, S. (2014) Universidad pública y experiencia estudiantil: de los estudios de caso a las agendas políticas de la educación superior. Universidades, (60),41-50 Disponible en: https://ri.conicet.gov.ar/bitstream/handle/11336/35807/CONICET Digital Nro.e8d6a192-2f79-40c1-8b50ba3ed4376284 B.pdf? sequence $=5$ \&isAllowed $=y$

- Carrizo, C., Cruz, F. Y Vásquez, O. (2017) Tutorías: un espacio de acompañamiento universitario. El rol del tutor de la carrera de Comunicación Social en la Facultad de Humanidades y Ciencias Sociales de la Universidad Nacional de Jujuy, año 2015. Revista: REVCOM; no. 4. Red de Carreras de Comunicación Social (REDCOM) Jujuy, Argentina. 
Disponible en: http://sedici.unlp.edu.ar/handle/10915/68111

- Chiavenato, I. (2000). Administración de recursos humanos. (5 Bogotá, Colombia. Ed. Mc. Graw Hill.,

- Decreto 366/2006. Convenio Colectivo de Trabajo para el Sector No Docente de las Instituciones Universitarias Nacionales. Disponible en: http://www.fatun.org.ar/fatun2 convenio colectivo.html

- Ehuletche, A. M. Y De Stefano, A. (2009) Competencias y capacidades en las tutorías virtuales. IV Congreso de Tecnología en Educación y Educación en Tecnología. Red de Universidades con Carreras en Informática (RedUNCI) Disponible en: http://sedici.unlp.edu.ar/handle/10915/18965

- García, R. I., Cuevas, O., Vales, J. J. y Cruz, I. (2012). Impacto del Programa de Tutoría en el desempeño académico de los alumnos del Instituto Tecnológico de Sonora. Revista Electrónica de Investigación Educativa, 14(1), 106-121. Disponible en http://redie.uabc.mx/vol14no1/contenido-garciaetal.html

- García Aretio L. (coord.) (2009) Concepción y tendencias de la educación a distancia en américa latina. Documentos de trabajo | № 02 Ed. Centro de Altos Estudios Universitarios de la OEI.

- García Aretio, L. (2007). ¿Educación presencial/no presencial? Boletín Electrónico de Educación a Distancia BENED, 1-5. Disponible en: http://espacio.uned.es/fez/eserv.php?pid=bibliuned:20093\&ds|D=educpr esenc.pdf

- González, A. Y Martin, M. M. (Comp.)( 2017) $4^{\circ}$ Jornadas de TIC e Innovación en el Aula. Más allá del aula virtual. "Otros horizontes, otros 
desafíos" Ed. Universidad Nacional de La Plata. Dirección de Educación a Distancia, Innovación en el aula y TIC (EAD).

Disponible en: http://sedici.unlp.edu.ar/handle/10915/65078

- Hernández Aguilar, L. (2012). Modelos Tutoriales en la Educación a Distancia a través de Tecnologías de la Información y la Comunicación: Tareas del docente tutor. Tesis Doctoral. Facultad de Educación. Universidad Nacional de Educación a Distancia. Madrid. España.

Disponible en: http://e-spacio.uned.es/fez/eserv/tesisuned:EducacionMlhernandez/Documento.pdf

- Hernando, G. (2001) Las prácticas de la enseñanza en la Educación Superior. Reflexiones pedagógico-didácticas sobre los usos de las tecnologías informáticas en las aulas. Facultad de Humanidades y Ciencias de la Educación. UNLP.

Disponible en: http://sedici.unlp.edu.ar/handle/10915/2946

- Guido, L. y Versino, M. (2010) La oferta académica 'virtual' en universidades nacionales argentinas y su expansión territorial: Un estudio de casos de 'campos virtuales'. Archivos de Ciencias de la Educación, 4a. época, 4(4). Disponible en http://www.memoria.fahce.unlp.edu.ar/art revistas/pr.4775/pr.4775 .pdf

- Lara Villanueva, S. (2005) El aprendizaje cooperativo: un modelo de intervención para los programas de tutoría escolar en el nivel superior. Revista. Educación Superior vol.34, n.133, pag.87-104. ISSN 0185-2760.

Disponible en http://www.scielo.org.mx/pdf/resu/v34n133/0185-2760resu-34-133-87.pdf 
- Lobato Fraile, C.y Guerra Bilbao, N (2016). La tutoría en la educación superior en Iberoamérica: Avances y desafíos. EDUCAR, 52(2),379-398. Disponible en: https://www.redalyc.org/pdf/3421/342146063009.pdf

- Ordenanza 262/02 de la UNLP. Ingreso y Ascenso del personal no docente de la UNLP. Disponible en: . http://sedici.unlp.edu.ar/handle/10915/25620

- Parrino, M.C. (2014) ¿Evasión o expulsión? Los mecanismos de la deserción universitaria. Buenos Aires, Argentina. Ed. Biblos.

- Quintero Cortés Y. (2017). Implementación de un programa de tutorías como estrategia de gestión académica, para mejorar el rendimiento académico de los estudiantes de inglés como lengua extranjera en un centro de idiomas. Colombia. Bogotá.

Disponible

en:

https://repository.unilibre.edu.co/bitstream/handle/10901/10315/TESIS.\% 20IMPLEMENTACIÓN\%20DE\%20UN\%20PROGRAMA\%20DE\%20TUT ORÍAS..pdf?sequence $=1$

- Perez Lindo, A. (2014). La formación del profesor del futuro. Publicado en: Caamaño C. (coord..) (2015) En busca de una docencia para nuestro tiempo. Montevideo: Magró, pp. 27 -42

- Perez Lindo, A. (2018) El uso social del conocimiento y la universidad. Buenos Aires, Argentina. Ed.Teseo

- Perrenoud .P. (2007) Desarrollar la practica reflexiva en el oficio de enseñar. Profesionalización y razón pedagógica. Introd. y cap. 1. DF, México. Ed. GRAÓ 
- Santos Sharpe, A. y Carli S. (2016) Estudios globales y locales sobre el abandono de los estudios universitarios. Teorías, perspectivas y nuevos abordajes. RAES ISSN 1852-8171 / Año 8/ Número 13.

Disponible en: http://www.revistaraes.net/revistas/raes13 art1.pdf

- Schön D. (1992) La Formación de Profesionales Reflexivos. Hacia un nuevo diseño de la enseñanza y el aprendizaje en las profesiones. Cap. 1 y 2.Barcelona. España. Ed. Paidós.

- Sistema de consulta de estadísticas universitaria. (2020) Secretaría de Políticas Universitarias de la Nación - SPU-

Disponible en: http://estadisticasuniversitarias.me.gov.ar/\#/home

- Sosa, R. E. (2011). Los saberes "necesarios": la producción y legitimación social. El rol de la universidad. Trabajo y Sociedad, $\mathrm{XV}(17), 201-212$.

- Disponible en: https://www.redalyc.org/articulo.oa?id=3873/387334689014

- Tauber, F. (2018) "Pensar la Universidad. Proyecto Institucional de la Universidad Nacional de La Plata. 2018-2022" (1 Ed).

- Disponible en:

http://sedici.unlp.edu.ar/bitstream/handle/10915/68411/Versi\%C3\%B3n 2. pdf? sequence $=2$ \&is Allowed $=y$

- Zampar, M. (2016) Análisis de estrategias de tutorías virtuales. Revisión de la utilización de herramientas asincrónicas (Tesis) Facultad de Informática. Universidad Nacional de La Plata.

Disponible en: http://sedici.unlp.edu.ar/handle/10915/56270 